infection by activating the transcription of anti-microbial genes. Accordingly, cells lacking the mammalian imd homologue, receptor interacting protein 1 (RIP) were also found to be defective in Type I IFN induction and antiviral activity in response to virus/dsRNA, similar to FADD deficient cells. This data indicate the existence of a key intracellular dsRNA/virus recognition pathway in mammalian cells, which is central for the induction Type I IFN and the activation of other important primary innate immune response genes.

In addition, our laboratory has recently shown that vesicular stomatitis virus, VSV, a relatively non-pathogenic, negative-stranded RNA virus, can selectively induce the cytolysis of malignant cells, through the induction of apoptotic cell death. VSV appears able to selectively replicate in transformed cells since these hosts exhibit the hallmarks of a flawed interferon (IFN) system, which is essential for preventing VSV replication. In part, these cellular flaws may include prevalent defects in the regulation of cellular translation. Wild type VSV causes significant tumor regression when administered at sites distal from the tumor, when delivered intravenously, or against syngeneic tumors in immunocompetent hosts. The simple genetic constitution of VSV, lack of any known transforming properties, well studied immunobiology and the ability to genetically manipulate this virus affords an ideal opportunity to further enhance the oncolytic potential of this generally innocuous organism. Accordingly, we have constructed recombinant VSVs that carried cytokines such as IL-4, IL-12 or the Type I or II interferons. We have determined that such viruses were not only viable but synthesized their heterologous products to extremely high levels. In addition, all engineered viruses exhibited greatly increased attenuation, more potent oncolytic activity against metastatic disease in immunocompetent animals than the wild-type virus and were able to stimulate specific antitumor CTL responses. Collectively, our data demonstrates that VSV expressing immunodulatory genes could provide a promising approach to cancer therapy and be useful tools for examining mechanisms of tumorigenesis.

\title{
Autologous Hematopoetic Stem Cell Transplantation for HIV-related non-Hodgkin's Lymphomas (NHL)
}

\author{
Henrique Bittencourt
}

Autologous hematopoetic stem cell transplantation (AHSCT) consist of a myeloablative chemotherapy followed by a rescue with autologous peripheral stem cell transplantation collected and cryopreserved before. The principle is to deliver a maximum tolerated dose of chemotherapy and rescue the bone marrow function with the infusion of hematopoetic stem cells. Data from the CIBMTR show that multiple myeloma, non-Hodgkin's lymphoma (NHL) and Hodgkin's disease (HD) are the most frequent indications of AHSCT in North America. Specifically for NHL, AHSCT is indicated for patients in second remission or in chemosensitive relapse. A randomized trial published in 1995 showed that AHSCT provided superior overall and disease-free survival when compared to salvage chemotherapy alone. (Philip T, N Engl J Med 1995)

NHL is the second most frequent cancer in HIV patients. It is usually very aggressive, with a worst prognostic compared to non-HIV-related NHL. Difuse large-cell lymphoma and small non-cleaved cell lymphoma comprised more that 95\% of the NHL in HIV-patients. Initial approach to treat HIV-related NHL using the same regimens employed in non-HIV patients. Toxicity was for more superior and results inferior. Next step was to decrease intensity of chemotherapy in order to reduce toxicity, maintaining the initial response rate. More recently, aggressive regimens (EPOCH, CDE) associated with HAART, improved response rate and survival, with acceptable toxicity. Nevertheless, more than half of patients eventually relapse. For this patients, salvage chemotherapy only, as in non-HIV patients, is only palliative and associated with high toxicity. Initial attempts using AHSCT for relapsed HIV-related NHL patients failed due to a high frequency of opportunistic infections and AIDS evolution.

Furthermore, collection of peripheral blood stem cell (PBSC) was very difficult, due to the use of AZT. More 
recently, with the availability of HAART, new approaches to relapsed HIV-related NHL were tried. A prospective trial was published in 2003 on JCO. Sixteen patients with HIV-related NHL or HD were treated sequentially by salvage chemotherapy, followed by mobilization of PBSC with cyclophosphamide and conditioning with BEAM (BCNU, etoposide, Ara-C and melphalan). Tem patients received the AHSCT, with 7 complete and 2 partial remissions. Six patients maintained remission after 8 months of follow-up. (Re A, et al. J Clin Oncol 2003) A retrospective study in France in relapsed HIV-related NHL showed that, after AHSCT, 10 out of 14 patients achieved complete remission. After a median follow-up of 25months, 5/15 patients were alive. (Gabarre J, et al. Haematologica 2004) Recently, the group from City of Hope published a trial where 20 patients with high-risk features, in relapse or refractory, with AHSCT. With a median follow-up of more than 2.5 years, 17 out of 20 were alive in remission and toxicity was low.(Krishnan A, et al. Blood 2005)

Recently trial listed above pointed to AHSCT as a valuable alternative to treat high-risk or relapsed HIVrelated NHL. Its results are far superior compared to salvage chemotherapy only. Nevertheless, this results must be taken with caution. This series are relatively small and non-controlled trials. A randomized and larger clinical trial is needed.

\section{Profiling viral gene expression in lymphomas}

\section{Dirk P. Dittmer}

One quarter of human cancers are associated with infectious agents such as viruses. Transcriptional profiling of the viral genome offers the chance to accelerate our investigations, diagnosis and staging of viral-associated lymphomas. Since viral genomes are orders of magnitude smaller than the human genome, we have developed whole viral genome arrays based upon real-time quantitative PCR for Kaposi's Sarcoma-associated herpesvirus and Epstein-Barr virus (1). This technology is technologically robust, rapid and inexpensive. Most clinical laboratories and research centers have extensive experience in real-time QPCR, which has become routine for HIV diagnostics and thuas are in a position to use QPCR-based arrays for lymphoma diagnosis. In adopting realtime QPCR to comparative transcription profiling for KSHV we realized that we could feed the real-time QPCR output (the so-called CT value) directly in existing publicly available cluster analysis programs (2). In fact, the initial step in hybridization-based analysis, e.g. Affymatrix is to compute the logarithm of the signal intensity in order to improve statistical performance. The CT values already represent a logarithmic measure of the target concentration and can be used directly. PCR is the most sensitive detection method available today. It is inherently more sensitive than hybridization-based detection methods and we have been able to quantify 96 different viral mRNAs from a 2x2 mm fine-needle KS biopsy or from as little as 5000 FACS sorted cells.

\section{EBV-Associated Lymphoma in Bahia, Brazil}

Iguaracyra Araujo, Achiléa Bittencourt, Helenemarie S. Barbosa, Tatiana Gil Portugal, Daniel Freitas, Daniela Almeida, Núbia Mendonça, Michael Hummel and Hans-Dieter Foss

Epstein-Barr virus (EBV) is a lymphotropic virus associated with some human malignancies such as endemic Burkitt's lymphoma, Hodgkin's lymphoma (HL), AIDS-associated lymphomas. We report the frequency of EBVinfection in pediatric Burkitt‘s lymphoma (BL), pediatric Hodgkin's lymphoma (HL), adulthood HL and AIDS related non-Hodgkin lymphoma (NHL) occurring in Bahia, Brazil. For comparison we described also the frequency of EBV infection in 28 lymphomas not related to HIV-infection and in 40 tonsils from children living in Bahia and 